\title{
EXPLICIT CONSTRUCTION OF UNIVERSAL OPERATOR ALGEBRAS AND APPLICATIONS TO POLYNOMIAL FACTORIZATION
}

\author{
DAVID P. BLECHER AND VERN I. PAULSEN
}

(Communicated by Paul S. Muhly)

\begin{abstract}
Using the characterization of unital operator algebras developed in [6], we give explicit internal definitions of the free product and the maximal operator-algebra tensor product of operator algebras and of the group operator algebra $\mathrm{OA}(G)$ of a discrete semigroup $G$ (if $G$ is a discrete group, then $\mathrm{OA}(G)$ coincides with the group $C^{*}$-algebra $\left.C^{*}(G)\right)$. This approach leads to new factorization theorems for polynomials in one and two variables.
\end{abstract}

\section{INTRODUCTION}

There are many constructions in the theory of operator algebras (selfadjoint or otherwise) which are described purely extrinsically, in terms of classes of representations of the object under consideration. Three examples of this are the maximal operator-algebra tensor product of two operator algebras [16], the free product (or coproduct) of $C^{*}$-algebras $[3,7,8]$, and the group $C^{*}$-algebra $C^{*}(G)$ of a discrete group $G$ [18]. In each of the above, the norm is defined as a supremum over a class of representations on Hilbert space; that is, we endow an appropriate algebra with a norm which will make the completion into an operator algebra with the correct universal property. Except in certain special cases (for instance, if the group $G$ is amenable), it has hitherto been impossible to describe these constructions in any other way. In this note we give explicit internal expressions for the norms. In addition, we define here the free product of (not necessarily selfadjoint) operator algebras and the semigroup operator algebra $\mathrm{OA}(G)$ of a discrete semigroup $G$.

As a consequence of our norm formulae, we obtain rather remarkable factorization theorems. For instance, we prove that, for a polynomial in two variables, the norm supremum over the bidisc is less than 1 if and only if the polynomial may be factored as a product of matrix-valued polynomials in a single variable each of which has a norm supremum over the disc that is less than 1.

Received by the editors January 22, 1990 and, in revised form, April 26, 1990.

1980 Mathematics Subject Classification (1985 Revision). Primary 47D25, 46L05; Secondary 46M05, $20 \mathrm{M} 30$.

This research was partially supported by the National Science Foundation. 
Recall that if $\mathscr{H}$ is a Hilbert space, then the space $M_{n}(B(\mathscr{H}))$ of $n \times n$ matrices with entries in $B(\mathscr{H})$ has a natural norm $\|\cdot\|_{n}$ via the identification of $M_{n}(B(\mathscr{H}))$ and $B\left(\mathscr{H}^{(n)}\right)$; if $\mathscr{A}$ is a subalgebra of $B(\mathscr{H})$, then by restricting $\|\cdot\|_{n}$ to $M_{n}(\mathscr{A})$ we obtain a sequence of norms $\left\{\|\cdot\|_{n}\right\}$ called the matrix norms of the operator algebra $\mathscr{A}$. We write $M_{n}$ for $M_{n}(\mathbf{C})$. More generally, we write $M_{m, n}$ for $m \times n$ matrices and $\|\cdot\|_{m, n}$ for the natural norm on $M_{m, n}(\mathscr{A})$. We shall assume a certain familiarity with the theory of matrix norms and completely bounded maps, as may be gleaned from [2, 15, 6, 9], for instance.

Throughout, by an "operator algebra" we shall mean an algebra of bounded operators on a Hilbert space together with its sequence of matrix norms or, more loosely, any algebra that can be represented as such. We do not require the algebra to be selfadjoint or uniformly closed. All operator algebras considered in this paper are unital (contain an identity of norm 1). We identify two operator algebras which are completely isometrically isomorphic-that is, if there is an algebraic isomorphism between them which preserves the matrix norms.

The main aim of this paper is to give expressions for the matrix norms of the three operator-algebra constructions mentioned in the first paragraph. Our results are obtained by the characterization of operator algebras given in [6]. This continues the program (see $[6,5]$ ) of studying operator-algebra constructions in terms of their matricial structure.

It will become clear that the methods of this paper should be valid for other categorical constructions. It is curious that even when the norms we write down are $C^{*}$-norms, it seems impossible to verify directly from the abstract $C^{*}$ condition that they are so: thus, in some situations it seems that the conditions of [6] are more useful than the G.N.S. characterization.

\section{THE SEMIGROUP OPERATOR ALGEBRA}

Because the group $C^{*}$-algebra $C^{*}(G)$ of a discrete group $G$ is well known, and because the calculations here are in some ways the clearest, we begin by describing this construction. We refer the reader to [18] for the usual (extrinsic) description of $C^{*}(G)$.

Throughout this paper all the semigroups considered have an identity.

Let $G$ be a discrete semigroup. By a contractive representation of $G$, we shall mean a unital homomorphism of $G$ into the semigroup of contraction operators on a Hilbert space. We define the semigroup operator algebra $\mathrm{OA}(G)$ of $G$ to be an operator algebra with the following universal property: there is a contractive representation $i$ of $G$ into $\mathrm{OA}(G)$ whose range generates $\mathrm{OA}(G)$, and if $\pi$ is a contractive representation of $G$ on a Hilbert space $\mathscr{H}$ then there is a unique completely contractive unital homomorphism $\pi^{\sim}$ from $\mathrm{OA}(G)$ into $B(\mathscr{H})$ such that $\pi^{\sim} \circ i=\pi$. We now show that $\mathrm{OA}(G)$ exists; clearly, if it does, then the universal property characterizes $\mathrm{OA}(G)$ up to unital complete isometric isomorphism.

We write $\mathbf{C} G$ for the semigroup algebra of $G$, that is, the set of formal 
linear combinations of elements of $G$ with the obvious algebra structure. Each contractive representation $\theta$ of $G$ induces a unital representation $\theta^{\sim}$ of the semigroup algebra $\mathbf{C} G$. We may define a seminorm $\|\cdot\|_{n}$ on $M_{n}(\mathbf{C} G)$ by

$$
\left\|\left[u_{i j}\right]\right\|_{n}=\sup \left\{\left\|\left[\theta^{\sim}\left(u_{i j}\right)\right]\right\|: \text { contractive representations } \theta \text { of } G\right\} .
$$

If $\mathscr{N}=\left\{u \in \mathbf{C} G:\|u\|_{1}=0\right\}$, then $\mathscr{N}$ is a linear subspace of $\mathbf{C} G$ and \|\|$\cdot \|_{n}$ induces a norm on $M_{n}(\mathbf{C} G / \mathscr{N})$ which we also write as $\|\cdot \cdot\|_{n}$. One may use a direct sum argument or the characterization in [6] to show that $\mathrm{C} G / \mathcal{N}$, together with the sequence of matrix norms $\left\{\|\| \cdot \|_{n}\right\}$, is an operator algebra. There is a contractive representation $i$ of $G$ into $\left(\mathbf{C} G / \mathscr{N},\|\cdot\|_{1}\right)$; of course, $i$ is a monomorphism if and only if there is a faithful contractive representation of $G$ on some Hilbert space. In any case it is clear that the operator algebra $\left(\mathbf{C} G / \mathcal{N},\|\| \|_{n}\right)$ has the correct universal property, and we may identify $\left(\mathbf{C} G / \mathscr{N}, \mid\|\cdot\| \|_{n}\right)$ and $\mathrm{OA}(G)$.

There are of course many semigroups (see [4] for a survey) for which $\mathscr{N}=$ $\{0\}$, and in the application that we give, this is always the case. For instance, if $G$ is a semigroup with left (right) cancellation then one may obtain a faithful representation of $\mathbf{C} G$ as follows: We may regard $G$ as an orthonormal basis of $1^{2}(G)$; then the left (right) regular representation of $G$ on itself extends to a representation $\theta$ of $G$ on $1^{2}(G)$. If $G$ has left (right) cancellation, then the range of $\theta$ consists of isometries. It is clear that $\theta$ extends to a faithful representation $\theta^{\sim}$ of $\mathbf{C} G$ on $1^{2}(G)$. The matrix norms on $\mathbf{C} G$ determined by $\theta$ are dominated by the matrix norms $\left\{\|\| \cdot \|_{n}\right\}$; thus $\|U\|_{n}=0$ implies $U=0$.

Proposition 2.1. If $G$ is a discrete group, then $\mathrm{OA}(G)$ possesses a natural involution. With respect to this involution, the norm closure of $\mathrm{OA}(G)$ is a $C^{*}$-algebra which coincides with the group $C^{*}$-algebra $C^{*}(G)$.

Proof. Any unital contractive representation of a group $G$ is a unitary representation of $G$, and hence extends to a unital *-homomorphism on $C^{*}(G)$. Thus $C^{*}(G)$ is completely isometrically isomorphic to the norm closure of $\mathrm{OA}(G)$. Under this isomorphism the involution on $C^{*}(G)$ corresponds to the natural involution on $\mathbf{C} G$.

The following examples, all of which are cancellative semigroups, are of interest to us:

Example 2.2. If $G=\left(\mathbf{N}_{\mathbf{0}},+\right)$, the natural numbers (including zero) with addition, then $\operatorname{OA}(G)$ is the universal operator algebra generated by a contraction. That is, it has the following property: given a contraction $T$ on a Hilbert space $\mathscr{H}$, then there is a completely contractive homomorphism $\pi$ from $\mathrm{OA}(G)$ into $B(\mathscr{H})$ such that $\pi(n)=T^{n}$ for each $n \in \mathbf{N}_{\mathbf{0}}$. It is a well-known consequence of Sz-Nagy's unitary dilation for contractions [20] that the universal (closed) operator algebra generated by a contraction is the disc algebra $A(\mathbf{D})$ completely isometrically. Thus the closure of $\mathrm{OA}\left(\left(\mathbf{N}_{\mathbf{0}},+\right)\right)$ is $A(\mathbf{D})$ via the map 
$n \rightarrow z^{n}$. Note that von Neumann's inequality [23] only identifies $\mathrm{OA}\left(\left(\mathbf{N}_{\mathbf{0}},+\right)\right)$ and $A(\mathbf{D})$ isometrically.

Example 2.3. If $G=\left(\mathbf{N}_{\mathbf{0}} \times \mathbf{N}_{\mathbf{0}},+\right)$, then $\mathrm{OA}(G)$ is the universal operator algebra generated by two commuting contractions. That is, it has the following property: given commuting contractions $T_{1}$ and $T_{2}$ on a Hilbert space $\mathscr{H}$, then there is a completely contractive homomorphism $\pi$ from $\mathrm{OA}(G)$ into $B(\mathscr{C})$ such that $\pi((n, m))=T_{1}^{n} T_{2}^{m}$ for each $n, m \in \mathbf{N}_{\mathbf{0}}$. It is a consequence of Ando's simultaneous unitary dilation theorem for two commuting contractions [1] that the universal (closed) operator algebra generated by two commuting contractions is the bidisc algebra $A(\mathbf{D} \times \mathbf{D})$, completely isometrically. Thus the closure of $\mathrm{OA}\left(\left(\mathbf{N}_{\mathbf{0}} \times \mathbf{N}_{\mathbf{0}},+\right)\right)$ is $A(\mathbf{D} \times \mathbf{D})$ via the map $(n, m) \rightarrow z^{n} w^{m}$.

Example 2.4. Little is known about $\mathrm{OA}\left(\mathbf{N}_{\mathbf{0}} \times \mathbf{N}_{\mathbf{0}} \times \mathbf{N}_{\mathbf{0}},+\right)$, the universal operator algebra generated by three commuting contractions, except that it is not the tridisc algebra $[10,22]$ and is not even a uniform algebra. Unlike the previous two examples, we are unable to say precisely what this operator algebra is. If $G=(\mathbf{N}, \times)$, the natural numbers with multiplication, then $\mathrm{OA}(G)$ is the universal operator algebra generated by countably many commuting contractions.

Remark. Given an operator algebra $\mathscr{A}$, we can form the universal $C^{*}$-algebra $C^{*}(\mathscr{A})$ generated by $\mathscr{A}$ : this has the property that every completely contractive unital homomorphism of $\mathscr{A}$ into a $C^{*}$-algebra $\mathscr{B}$ extends to a *homomorphism of $C^{*}(\mathscr{A})$ into $\mathscr{B}$. For a semigroup $G$, we define $\operatorname{STAR}(G)$ to be the universal *-semigroup generated by $G$; that is, $\operatorname{STAR}(G)$ is the unique ${ }^{*}$-semigroup containing $G$ as a subsemigroup with the following universal property: every unital semigroup homomorphism of $G$ into a *-semigroup $H$ extends to a *-homomorphism from $\operatorname{STAR}(G)$ into $H$. If $G^{o p}$ is $G$ with reversed multiplication, then one may regard $\operatorname{STAR}(G)$ as the free product $G^{o p} * G$, with the obvious involution. Then we have $C^{*}(\operatorname{OA}(G))=C^{*}(\operatorname{STAR}(G))$, where $C^{*}(\operatorname{STAR}(G))$ is the enveloping $C^{*}$-algebra of the Banach *-algebra $1^{1}(\operatorname{STAR}(G))$. Indeed, both of these $C^{*}$-algebras are equal to the universal $C^{*}$-algebra generated by $G$. It is clear that if $G=\left(\mathbf{N}_{\mathbf{0}},+\right)$, then $C^{*}(\mathrm{OA}(G))$ is the $C^{*}$-algebra generated by a universal contraction studied in [14, 13], and elsewhere.

We now proceed to define $\mathrm{OA}(G)$ intrinsically. We define for a matrix $U$ in $M_{n}(\mathbf{C} G)$ the following quantity:

$$
\|U\|_{n}=\inf \left\{\left\|A_{0}\right\|\left\|A_{1}\right\| \cdots\left\|A_{m}\right\|\right\},
$$

where the infimum is taken over all natural numbers $m$ and $k$ and all ways to write $U=A_{0} G_{1} A_{1} \cdots G_{m} A_{m}$, with $A_{0} \in M_{n, k}, A_{m} \in M_{k, n}$, and $A_{i} \in M_{k}$ for $i=1, \ldots, m-1$, and with each $G_{i}$ a $k \times k$ diagonal matrix with entries in $G$. This quantity is easily seen to be the same as the infimum taken over all ways to write $U=A_{0} G_{1} A_{1} \cdots G_{m} A_{m}$, where the $A_{i}$ are rectangular matrices of any size, and the $G_{i}$ are square diagonal matrices of any size, so that their product 
makes sense. It is not hard to see that any $U$ in $M_{n}(\mathbf{C} G)$ can be expressed in such a way (in fact with $m=3$ ), and so $\|\cdot\|_{n}$ is well defined.

Lemma 2.5. The quantities $\|\cdot\|_{n}$ are seminorms which dominate $\|\cdot\|_{n}$. Moreover, for $\left[u_{i j}\right] \in M_{n}(\mathbf{C} G)$, we have $\left\|\left[u_{i j}\right]\right\|_{n}=0$ if and only if $\left\|u_{i j}\right\|_{1}=0$ for all $i, j=1, \ldots, n$.

Proof. This is similar to the corresponding calculation for the Haagerup norm; see $[11,17]$. Let $U, V \in M_{n}(\mathbf{C} G)$, with $U=A_{0} G_{1} A_{1} \cdots G_{m} A_{m}$ and $V=$ $B_{0} H_{1} B_{1} \cdots H_{m} B_{m}$ (by adding on identity matrices it is clear that we may assume that the "lengths" of the representations are the same). Without loss of generality, we may assume

$$
\left\|A_{1}\right\|=\left\|A_{2}\right\|=\cdots=\left\|A_{m-1}\right\|=\left\|B_{1}\right\|=\left\|B_{2}\right\|=\cdots=\left\|B_{m-1}\right\|=1,
$$

and that $\left\|A_{0}\right\|=\left\|A_{m}\right\|$ and $\left\|B_{0}\right\|=\left\|B_{m}\right\|$. Then

$$
U+V=\left[A_{0} B_{0}\right]\left(G_{1} \oplus H_{1}\right)\left(A_{1} \oplus B_{1}\right) \cdots\left(G_{m} \oplus H_{m}\right)\left[A_{m}^{t} B_{m}^{t}\right]^{t},
$$

and so

$$
\begin{aligned}
\|U+V\|_{n} & \leq\left\{\left\|A_{0}\right\|^{2}+\left\|B_{0}\right\|^{2}\right\}^{1 / 2}\left\{\left\|A_{m}\right\|^{2}+\left\|B_{m}\right\|^{2}\right\}^{1 / 2} \\
& =\left\|A_{0}\right\|\left\|A_{m}\right\|+\left\|B_{0}\right\|\left\|B_{m}\right\| \\
& =\left\|A_{0}\right\|\left\|A_{1}\right\| \cdots\left\|A_{m}\right\|+\left\|B_{0}\right\|\left\|B_{1}\right\| \cdots\left\|B_{m}\right\|,
\end{aligned}
$$

which shows that $\|U+V\|_{n} \leq\|U\|_{n}+\|V\|_{n}$. Thus $\|\cdot\|_{n}$ is a seminorm.

The second assertion follows after applying a unital representation $\theta^{\sim}$ of the semigroup algebra $\mathbf{C} G$ determined by a contractive representation $\theta$ of $G$ to an element $U=A_{0} G_{1} A_{1} \cdots G_{m} A_{m} \in M_{n}(\mathbf{C} G)$.

Write $e_{i}$ for the usual basis of $\mathbf{C}^{n}$ and $e_{i j}$ for the matrix units in $M_{n}$. Then for $\left[u_{i j}\right] \in M_{n}(\mathbf{C} G)$, we have $u_{k l}=e_{k}^{t}\left[u_{i j}\right] e_{l}$, so that $\left\|u_{k l}\right\|_{1} \leq\left\|\left[u_{i j}\right]\right\|_{n}$. Also $\left\|\left[u_{i j}\right]\right\|_{n}=\left\|\sum_{i, j} e_{i i}\left[u_{i j}\right] e_{j j}\right\|_{n} \leq \sum_{i, j}\left\|e_{i i}\left[u_{i j}\right] e_{j j}\right\|_{n} \leq \sum_{i, j}\left\|u_{i j}\right\|_{1}$, which proves the final assertion.

Let $\mathscr{M}=\left\{u \in \mathbf{C} G:\|u\|_{1}=0\right\}$. Then, by the lemma, $\mathscr{M}$ is a linear subspace of $\mathbf{C} G$, and $\mathscr{N} \supset \mathscr{M}$. By the third assertion of the lemma, $\|\cdot\|_{n}$ induces a norm on $M_{n}(\mathbf{C} G / \mathscr{M})$, which we also write as $\|\cdot\|_{n}$. Of course if $\mathscr{N}=\{0\}$, then $\mathscr{M}=\{0\}$, and this is the case we are interested in for the applications.

Theorem 2.6. The algebra $\mathrm{C} G / \mathscr{M}$, together with the matrix norms $\|\cdot\|_{n}$, is an operator algebra.

Proof. We use the characterization of an operator algebra given in [6]. For elements $U, V \in M_{n}(\mathbf{C} G)$, it is clear that $\|U V\|_{n} \leq\|U\|_{n}\|V\|_{n}$, so it only remains to verify the $\left(L^{\infty}\right)$ condition $\|U \oplus V\|_{p+q}=\max \left\{\|U\|_{p},\|V\|_{q}\right\}$ for elements $U \in M_{p}(\mathbf{C} G)$ and $V \in M_{q}(\mathbf{C} G)$. Suppose $\varepsilon>0$ is given and we write $U=A_{0} G_{1} A_{1} \cdots G_{m} A_{m}$ and $V=B_{0} H_{1} B_{1} \cdots H_{m} B_{m}$ as before, but with $\left\|A_{1}\right\|=\left\|A_{2}\right\|=\cdots=\left\|A_{m}\right\|=\left\|B_{1}\right\|=\left\|B_{2}\right\|=\cdots=\left\|B_{m}\right\|=1,\left\|A_{0}\right\|<\|U\|_{p}+\varepsilon$, and $\left\|B_{0}\right\|<\|V\|_{q}+\varepsilon$. Then

$$
U \oplus V=\left(A_{0} \oplus B_{0}\right)\left(G_{1} \oplus H_{1}\right) \cdots\left(G_{m} \oplus H_{m}\right)\left(A_{m} \oplus B_{m}\right),
$$


and so

$$
\begin{aligned}
\|U \oplus V\|_{p+q} & \leq\left\|A_{0} \oplus B_{0}\right\| \cdots\left\|A_{m} \oplus B_{m}\right\| \\
& =\max \left\{\left\|A_{0}\right\|,\left\|B_{0}\right\|\right\} \\
& \leq \max \left\{\|U\|_{p}+\varepsilon,\|V\|_{q}+\varepsilon\right\},
\end{aligned}
$$

which proves that $\|U \oplus V\|_{p+q} \leq \max \left\{\|U\|_{p},\|V\|_{q}\right\}$. Finally, if $U \oplus V$ is written as $C_{0} K_{1} C_{1} \cdots K_{m} C_{m}$, then $U=[I 0] C_{0} K_{1} C_{1} \cdots K_{m} C_{m}[I 0]^{t}$, and so $\|U\|_{p} \leq\left\|[I 0] C_{0}\right\|\left\|C_{1}\right\| \cdots\left\|C_{m-1}\right\|\left\|C_{m}\left[\begin{array}{ll}I & 0\end{array}\right]^{t}\right\| \leq\left\|C_{0}\right\| \cdots\left\|C_{m}\right\|$, which shows that $\|U\|_{p} \leq\|U \oplus V\|_{p+q}$. Similarly, $\|V\|_{p} \leq\|U \oplus V\|_{p+q}$, and so $\|U \oplus V\|_{p+q}=$ $\max \left\{\|U\|_{p},\|V\|_{q}\right\}$.

Theorem 2.7. If $G$ is a discrete semigroup then $\mathscr{N}=\mathscr{M}$ and $\|\cdot\|_{n}=\|\cdot\| \|_{n}$ (as norms or as seminorms). Thus the semigroup operator algebra $\mathrm{OA}(G)$ of $G$ is the operator algebra $\left(\mathrm{C} G / \mathscr{M},\|\cdot\|_{n}\right)$.

Proof. Since $\left(\mathbf{C} G / \mathscr{M},\|\cdot\|_{n}\right)$ is an operator algebra, the canonical map $j$ from $G$ into $\mathrm{C} G / \mathscr{M}$ is a contractive representation of $G$ and extends to a completely contractive unital representation $j^{\sim}$ from $\left(\mathbf{C} G / \mathscr{N},\left|\|\cdot \mid\|_{n}\right)\right.$ into $(\mathbf{C} G / \mathscr{M}$, $\left.\|\cdot\|_{n}\right)$. Thus

$$
\left\|\left[u_{i j}\right]\right\|_{n}=\left\|\left[u_{i j}+\mathscr{M}\right]\right\|_{n}=\left\|\left[j^{\sim}\left(u_{i j}+\mathscr{N}\right)\right]\right\|_{n} \leq\left\|\left[u_{i j}+\mathscr{N}\right]\right\|_{n}=\|\left[\left[u_{i j}\right]\|\|_{n},\right.
$$

for $\left[u_{i j}\right] \in M_{n}(\mathbf{C} G)$, which together with the lemma shows that $\mathscr{N}=\mathscr{M}$ and that $\|\cdot\|_{n}=\|\| \cdot \mid \|_{n}$.

Corollary 2.8. If $G$ is a discrete group, then the completion of $\left(\mathbf{C} G,\|\cdot\|_{n}\right)$ is the group $C^{*}$-algebra $C^{*}(G)$. In particular, the norm on $C^{*}(G)$ is given explicitly by $\|\cdot\|_{1}$.

The theorem above has surprising geometrical applications. The observations contained in examples 2.2 and 2.3, together with the internal characterizations of the matrix norms on $\mathrm{OA}(G)$, lead to the following factorization theorems:

Corollary 2.9. Let $P(z)=\left[p_{i j}(z)\right]$ be a matrix-valued polynomial. Then $\sup \left\{\left\|\left[p_{i j}(z)\right]\right\|: z \in \mathbf{D}\right\}<1$ if and only if there exists a positive integer $m$ such that $P(z)$ can be factored as

$$
P(z)=A_{0} D_{1}(z) A_{1} D_{2}(z) \cdots D_{m}(z) A_{m},
$$

where the $A_{0}, \ldots, A_{m}$ are scalar matrices, each of norm less than 1 , and $D_{1}(z)$, $\ldots, D_{m}(z)$ are diagonal matrices with powers of $z$ as the diagonal entries.

Corollary 2.10. Let $P(z, w)=\left[p_{i j}(z, w)\right]$ be a matrix-valued polynomial. Then $\sup \left\{\left\|\left[p_{i j}(z, w)\right]\right\|: z, w \in \mathbf{D}\right\}<1$ if and only if there exists a positive integer $m$ such that $P(z, w)$ can be factored as

$$
P(z, w)=A_{0} D_{1}(z, w) A_{1} D_{2}(z, w) \cdots D_{m}(z, w) A_{m},
$$


where the $A_{0}, \ldots, A_{m}$ are scalar matrices, each of norm less than 1, and $D_{1}(z, w), \ldots, D_{m}(z, w)$ are diagonal matrices with monomials (terms of form $\left.z^{p} w^{q}\right)$ as the diagonal entries.

Remark. Corollaries 2.9 and 2.10 are equivalent to Sz-Nagy's and Ando's theorems, respectively. It is therefore possible that they may yield analytic proofs of these theorems.

By the remarks in 2.4 , the corresponding result for polynomials in three variables does not hold. However, this method gives

Corollary 2.11. For any positive integer $k$ and any matrix-valued polynomial $p$ in $k$ variables, we have

$$
\begin{aligned}
& \sup \left\{\left\|p\left(T_{1}, \ldots, T_{k}\right)\right\|: \text { all } k \text {-tuples of commuting contractions } T_{1}, \ldots, T_{k}\right\} \\
& \quad=\inf \left\{\left\|A_{0}\right\|\left\|A_{1}\right\| \cdots\left\|A_{m}\right\|\right\},
\end{aligned}
$$

where the infimum is taken over positive integers $m$, and all ways to write

$$
P\left(z_{1}, \ldots, z_{k}\right)=A_{0} D_{1}\left(z_{1}, \ldots, z_{k}\right) A_{1} D_{2}\left(z_{1}, \ldots, z_{k}\right) \cdots D_{m}\left(z_{1}, \ldots, z_{k}\right) A_{m},
$$

where $A_{0}, \ldots, A_{m}$ are scalar matrices and the $D_{i}\left(z_{1}, \ldots, z_{k}\right)$ are diagonal matrices with monomials as the diagonal entries.

\section{THE MAXIMAL OPERATOR ALGEBRA TENSOR PRODUCT}

We recall [16] that, if $\mathscr{A}$ and $\mathscr{B}$ are unital operator algebras, then one may define the maximal operator-algebra norm $\|\cdot\|_{\max }$ on the algebraic tensor product $\mathscr{A} \otimes \mathscr{B}$ as follows: If $\pi$ and $\theta$ are completely contractive representations of $\mathscr{A}$ and $\mathscr{B}$, respectively, on a Hilbert space $\mathscr{H}$, with commuting ranges, then we may define a representation $\pi \otimes \theta$ of $\mathscr{A} \otimes \mathscr{B}$ by

$$
(\pi \otimes \theta)(a \otimes b)=\pi(a) \theta(b),
$$

for $a \in \mathscr{A}$ and $b \in \mathscr{B}$. For $U=\left[u_{i j}\right] \in M_{n}(\mathscr{A} \otimes \mathscr{B})$, we define

$$
\|U\|_{\max }=\sup \left\{\left\|\left[(\pi \otimes \theta)\left(u_{i j}\right)\right]\right\|\right\},
$$

where the supremum is taken over all Hilbert spaces $\mathscr{H}$ and all pairs $\pi$ and $\theta$ of completely contractive representations of $\mathscr{A}$ and $\mathscr{B}$, respectively, on $\mathscr{H}$ with commuting ranges. If $\mathscr{A}$ and $\mathscr{B}$ are $C^{*}$-algebras, then this coincides with the projective $C^{*}$-tensor norm [21], and in this case there is an alternative way of describing the norm in terms of states [12], but again no purely internal descriptions as far as we know. We now proceed to describe this norm internally; most of the calculation follows the method of the previous section, so we shall be brief.

Following the notation of [17], if $A=\left[a_{i j}\right] \in M_{n, m}(\mathscr{A})$, then we write $A \otimes 1$ for the matrix $\left[a_{i j} \otimes 1\right]$ in $M_{n, m}(\mathscr{A} \otimes \mathscr{B})$; similarly $1 \otimes B$ has the obvious meaning. If $A_{1} \in M_{n, k}(\mathscr{A})$ and $A_{2}, \ldots, A_{m} \in M_{k}(\mathscr{A})$, and if $B_{m} \in$ $M_{k, n}(\mathscr{A})$, and $B_{1}, \ldots, B_{m-1} \in M_{k}(\mathscr{A})$, then the product

$$
\left(A_{1} \otimes 1\right)\left(1 \otimes B_{1}\right)\left(A_{2} \otimes 1\right)\left(1 \otimes B_{2}\right) \cdots\left(A_{m} \otimes 1\right)\left(1 \otimes B_{m}\right)
$$


is an element of $M_{n}(\mathscr{A} \otimes \mathscr{B})$. Any element of $M_{n}(\mathscr{A} \otimes \mathscr{B})$ may be written in such a way $[11,17]$ (indeed, with $m=2$ ). For $U \in M_{n}(\mathscr{A} \otimes \mathscr{B})$, define a quantity

$$
\|U\|_{n}=\inf \left\{\left\|A_{1}\right\|\left\|B_{1}\right\|\left\|A_{2}\right\| \cdots\left\|A_{m}\right\|\left\|B_{m}\right\|\right\}
$$

taken over all positive integers $m$ and over all ways to write $U$ in the form described above. This quantity is the same as the infimum taken over all ways to write

$$
U=\left(A_{1} \otimes 1\right)\left(1 \otimes B_{1}\right)\left(A_{2} \otimes 1\right)\left(1 \otimes B_{2}\right) \cdots\left(A_{m} \otimes 1\right)\left(1 \otimes B_{m}\right),
$$

where $A_{1}$ is an $n \times k_{1}$ matrix with entries in $\mathscr{A}, B_{1}$ is a $k_{1} \times k_{2}$ matrix with entries in $\mathscr{B}, A_{2}$ is a $k_{2} \times k_{3}$ matrix with entries in $\mathscr{A}$, and so on.

Theorem 3.1. The quantities $\|\cdot\|_{n}$ are norms, and $\mathscr{A} \otimes \mathscr{B}$ together with these matrix norms is an operator algebra.

Proof. By applying a representation $\pi \otimes \theta$ to an expression

$$
\left(A_{1} \otimes 1\right)\left(1 \otimes B_{1}\right) \cdots\left(A_{m} \otimes 1\right)\left(1 \otimes B_{m}\right),
$$

it is clear that the maximal operator-algebra tensor-product matrix norms on $\mathscr{A} \otimes \mathscr{B}$ are dominated by the matrix norms $\left\{\|\cdot\|_{n}\right\}$. Thus $\|U\|_{n}=0$ implies that $U=0$. The remainder of the proof follows the argument in $\S 2$.

Theorem 3.2. The maximal operator-algebra tensor-product matrix norms on $\mathscr{A} \otimes \mathscr{B}$ coincide with the matrix norms $\left\{\|\cdot\|_{n}\right\}$. In particular, if $\mathscr{A}$ and $\mathscr{B}$ are $C^{*}$-algebras, then the projective $C^{*}$-tensor norm $\|\cdot\|_{\max }=\|\cdot\|_{1}$ on $\mathscr{A} \otimes \mathscr{B}$. Proof. From Theorem 3.1 we see $\mathscr{A} \otimes \mathscr{B}$ with the matrix norms $\left\{\|\cdot\|_{n}\right\}$ is an operator algebra, and consequently has a completely isometric representation. Thus the matrix norms $\left\{\|\cdot\|_{n}\right\}$ are dominated by the matrix norms from the maximal operator algebra tensor product [16], and are consequently equal by the first sentence of the proof of Theorem 3.1.

Corollary 3.3. Let $P(z, w)=\left[p_{i j}(z, w)\right]$ be an $n \times n$ matrix-valued polynomial. Then $\sup \{\|P(z, w)\|: z, w \in \mathbf{D}\}<1$ if and only if the following condition is met: there exists a positive integer $m$, and matrices $P_{1}, \ldots, P_{m}, Q_{1}, \ldots, Q_{m}$, whose entries are polynomials in one variable, such that $\sup \left\{\left\|P_{i}(z)\right\|: z \in \mathbf{D}\right\}<1$ and $\sup \left\{\left\|Q_{i}(z)\right\|: z \in \mathbf{D}\right\}<1$ for each $i=1, \ldots, m$, and such that

$$
P(z, w)=P_{1}(z) Q_{1}(w) P_{2}(z) \cdots Q_{m}(w) .
$$

We may suppose that all the matrices above are $k \times k$ matrices for some positive integer $k$, except $P_{1}$ which is $n \times k$ and $Q_{m}$ which is $k \times n$.

Proof. This follows directly from the theorem above and Ando's theorem ([1] and [16, Proposition 2.5]) that the bidisk algebra $A(\mathbf{D} \times \mathbf{D})$ is completely isometrically isomorphic to $A(\mathbf{D}) \bar{\otimes}_{\max } A(\mathbf{D})$. 
Remark. Corollary 3.3 also follows immediately from Corollary 2.10. It is also equivalent to Ando's theorem.

\section{THE FREE PRODUCT OF OPERATOR ALGEBRAS}

The free product of $C^{*}$-algebras has been studied by many authors ([3], [5], [8],[24], and others) but still remains mysterious. We discuss here the so-called "biggest" free-product construction, or coproduct, and not "small" or spatial free products.

Let $\mathscr{A}$ and $\mathscr{B}$ be unital operator algebras, and let $\mathscr{A}{ }^{*} \mathscr{C} \mathscr{B}$ denote their algebraic free product amalgamated over the identity (in fact, the same approach is applicable in the case of amalgamation over any common unital subalgebra, but for simplicity we stick to the scalar case). This is the unique unital algebra $\mathscr{A} *{ }_{\mathrm{C}} \mathscr{B}$, which has the following universal property: there are unital imbeddings $i$ and $j$ of $\mathscr{A}$ and $\mathscr{B}$ respectively into $\mathscr{A}{ }^{*} \mathrm{C} \mathscr{B}$ such that the images of $\mathscr{A}$ and $\mathscr{B}$ under $i$ and $j$ respectively generate $\mathscr{A} *_{\mathrm{C}} \mathscr{B}$, and if $\pi$ and $\theta$ are unital homomorphisms from $\mathscr{A}$ and $\mathscr{B}$, respectively, into a third algebra $\mathscr{C}$, then there is a unique unital homomorphism $\pi * \theta$ of $\mathscr{A} *_{\mathrm{C}} \mathscr{B}$ into $\mathscr{C}$ with $(\pi * \theta) \circ i=\pi$ and $(\pi * \theta) \circ j=\theta$. It is folklore (and can be deduced from a result in [3]) that if $\mathscr{A}$ and $\mathscr{B}$ are unital $C^{*}$-algebras, then the algebraic amalgamated free product is a * -algebra with a faithful ${ }^{*}$-representation on Hilbert space.

We define the amalgamated free-product operator algebra, which we also denote as $\mathscr{A} *_{\mathrm{C}} \mathscr{B}$, to be the operator algebra which has the same universal property but with the imbeddings $i$ and $j$ complete isometric isomorphisms, the algebra $\mathscr{C}$ an operator algebra, and the maps $\pi$ and $\theta$ unital completely contractive homomorphisms. We need to prove that such an algebra exists. If it does, it is obviously unique up to complete isometric isomorphism. For convenience, in what follows, we identify $\mathscr{A}$ and $\mathscr{B}$ with subalgebras of $\mathscr{A}{ }^{*} \mathrm{C} \mathscr{B}$ and write, for instance, $a b$ for the element $i(a) j(b)$, if $a \in \mathscr{A}$ and $b \in \mathscr{B}$.

For $U \in M_{n}\left(\mathscr{A} *_{\mathrm{C}} \mathscr{B}\right)$, define a quantity

$$
\|U\|_{n}=\inf \left\{\left\|A_{1}\right\|\left\|B_{1}\right\|\left\|A_{2}\right\| \cdots\left\|A_{m}\right\|\left\|B_{m}\right\|\right\}
$$

taken over all positive integers $m$ and over all ways to write

$$
U=A_{1} B_{1} A_{2} B_{2} \cdots A_{m} B_{m},
$$

where $A_{1}$ is an $n \times k_{1}$ matrix with entries in $\mathscr{A}, B_{1}$ is a $k_{1} \times k_{2}$ matrix with entries in $\mathscr{B}, A_{2}$ is a $k_{2} \times k_{3}$ matrix with entries in $\mathscr{A}$, and so on.

Theorem 4.1. The quantities $\|\cdot\|_{n}$ are well defined norms, and $\mathscr{A} *_{\mathrm{C}} \mathscr{B}$, together with these matrix norms, is an operator algebra.

Proof. First we need to see that each $U \in M_{n}\left(\mathscr{A}{ }^{*} \mathrm{C} \mathscr{B}\right)$ has a representation of the type described above.

If $u \in \mathscr{A}{ }^{*} \mathrm{C} \mathscr{B}$, then $u$ has a representation as a sum of words formed by alternatively juxtaposing elements of $\mathscr{A}$ and $\mathscr{B}$. By adding 1's we may ensure 
that all the words have the same length. Thus $u$ has a representation which may be considered as an element of the tensor product $\mathscr{A} \otimes \mathscr{B} \otimes \mathscr{A} \otimes \cdots \mathscr{A} \otimes \mathscr{B}$ of a finite number of copies of $\mathscr{A}$ and $\mathscr{B}$. So if $U \in M_{n}\left(\mathscr{A} *_{\mathrm{C}} \mathscr{B}\right)$, then $U$ has a representation which may be considered as an element of the space $M_{n}(\mathscr{A} \otimes \mathscr{B} \otimes \mathscr{A} \otimes \cdots \mathscr{A} \otimes \mathscr{B})$. The fact that $U$ has a representation $U=$ $A_{1} B_{1} A_{2} B_{2} \cdots A_{m} B_{m}$ now follows from the analogous result for tensor products $[11,17]$.

Suppose $\mathscr{A}$ and $\mathscr{B}$ are unital subalgebras of $C^{*}$-algebras $\mathscr{A}_{1}$ and $\mathscr{B}_{1}$, and let $\mathscr{A}_{1}{ }^{\bar{*}} \mathscr{B}_{1}$ be the amalgamated free-product $C^{*}$-algebra of $\mathscr{A}_{1}$ and $\mathscr{B}_{1}$. This contains $\mathscr{A}{ }^{*} \mathscr{C} \mathscr{B}$ as a unital subalgebra in a 1-to-1 fashion. If $\pi$ and $\theta$ are $*$-representations of $\mathscr{A}_{1}$ and $\mathscr{B}_{1}$, respectively, on the same Hilbert space, then $\left\|(\pi * \theta)_{n}\left(A_{1} B_{1} \cdots A_{m} B_{m}\right)\right\| \leq\left\|A_{1} B_{1} \cdots A_{m} B_{m}\right\|_{n}$, and so it is clear that the amalgamated free-product $C^{*}$-algebra matrix norms restricted to $\mathscr{A}{ }^{*} \mathrm{C} \mathscr{B}$ are dominated by the matrix norms $\left\{\|\cdot\|_{n}\right\}$; thus, $\|U\|_{n}=0$ implies that $U=0$. The remainder of the proof is as in $\S 2$.

Theorem 4.2. If $\mathscr{A}$ and $\mathscr{B}$ are operator algebras, then the operator algebra $\left(\mathscr{A} *_{\mathrm{C}} \mathscr{B},\|\cdot\|_{n}\right)$ is the amalgamated free-product operator algebra; that is, it has the universal property described above. If $\mathscr{A}$ and $\mathscr{B}$ are $C^{*}$-algebras, then the amalgamated free-product $C^{*}$-algebra matrix norms on the algebraic free product $\mathscr{A} *_{\mathrm{C}} \mathscr{B}$ coincide with the matrix norms $\left\{\|\cdot\|_{n}\right\}$.

The proof of the above theorem is just as in the previous sections.

Finally we give some brief generalities about free-product operator algebras.

4.3. The nonamalgamated free product $\mathscr{A} * \mathscr{B}$ of unital operator algebras $\mathscr{A}$ and $\mathscr{B}$ may be described as follows: Let $\mathscr{A}^{1}$ and $\mathscr{B}^{1}$ be the operator algebras obtained by adding an identity to each algebra. (It is not hard to see that there is a unique way to extend the matrix norm structure to the unitizations which makes them again operator algebras.) Then $\mathscr{A} * \mathscr{B}$ may be identified completely isometrically with the codimension-1 subalgebra of $\mathscr{A}^{1}{ }_{\mathrm{C}} \mathscr{B}^{1}$ generated by $\mathscr{A}$ and $\mathscr{B}$. This has the desired universal property (the same statement as for the amalgamated free product but dropping the word "unital" throughout). This is because a completely contractive homomorphism from a unital operator algebra into $B(\mathscr{H})$ extends to a unital completely contractive homomorphism between the unitizations.

4.4. It follows immediately from our formulas from $\delta \S 3$ and 4 that the completed maximal operator algebra tensor product of two unital operator algebras is completely isometrically isomorphic to a quotient of the completed amalgamated free product of the algebras.

4.5. It is possible to see from the above theorem and the expression given in $\S 2$ that $\mathrm{OA}\left(G_{1}\right){ }_{\mathrm{C}} \mathrm{OA}\left(G_{2}\right)=\mathrm{OA}\left(G_{1} * G_{2}\right)$ for discrete semigroups $G_{1}$ and $G_{2}$. Of course, this also follows directly from the universal properties.

4.6. The amalgamated free product $\mathscr{A} *_{\mathrm{C}} \mathscr{B}$ of unital operator algebras $\mathscr{A}$ and $\mathscr{B}$ is completely isometrically isomorphic to the obvious subalgebra of 
the amalgamated free product $C^{*}(\mathscr{A}){ }_{\mathrm{C}} C^{*}(\mathscr{B})$ of the universal $C^{*}$-algebras which they generate.

4.7. F. Boca has proved [25] that if $\Phi$ and $\Psi$ are unital completely positive maps from $C^{*}$-algebras $\mathscr{A}$ and $\mathscr{B}$, respectively, into $B(\mathscr{H})$, then they have a common completely positive extension $\Phi{ }_{{ }_{\mathrm{C}}} \Psi$ from $\mathscr{A} *_{\mathrm{C}} \mathscr{B}$ into $B(\mathscr{H})$. Using this, together with the extension theorem for completely bounded maps and the fact that a unital completely contractive map on a $C^{*}$-algebra is completely positive, it follows that 4.6 above is true with $C^{*}(\mathscr{A})$ and $C^{*}(\mathscr{B})$ replaced by any containing $C^{*}$-algebra. Thus, the free product is completely injective; that is, if $\mathscr{A}_{1}$ and $\mathscr{B}_{1}$ are unital subalgebras of unital operator algebras $\mathscr{A}_{2}$ and $\mathscr{B}_{2}$, respectively, then $\mathscr{A}_{1}{ }{ }_{\mathrm{C}} \mathscr{B}_{1}$ is a unital subalgebra of $\mathscr{A}_{2}{ }_{\mathrm{C}} \mathscr{B}_{2}$ completely isometrically. The same statement with obvious modifications holds for the nonamalgamated free product of unital operator algebras. Now one can see that Boca's result generalizes to operator algebras: if $\mathscr{A}$ and $\mathscr{B}$ are unital operator algebras, and if $\Phi$ and $\Psi$ are unital completely contractive linear maps, then there is a common completely contractive linear extension to $\mathscr{A} *_{\mathrm{C}} \mathscr{B}$. We do not see how to deduce this last result directly from our characterization of the norm.

\section{REFERENCES}

1. T. Ando, On a pair of commuting contractions, Acta Sci. Math. 24 (1963), 88-90.

2. W. B. Arveson, Subalgebras of $C^{*}$-algebras. I, Acta Math. 123 (1969), 142-224.

3. D. Avitsour, Free products of $C^{*}$-algebras, Trans. Amer. Math. Soc. 271 (1982), 423-435.

4. B. A. Barnes, Representations of the $1^{1}$-algebra of an involutive semigroup, Trans. Amer. Math. Soc. 218 (1976), 361-396.

5. D. P. Blecher, Commutativity in operator algebras, Proc. Amer. Math. Soc. 109 (1990), 709-715.

6. D. P. Blecher, Z-J. Ruan, and A. M. Sinclair, A characterization of operator algebras, J. Funct. Anal. 89 (1990), 188-201.

7. L. G. Brown, Ext of certain free product algebras, J. Operator Theory 6 (1981), 135-141.

8. E. Christensen, E. G. Effros, and A. M. Sinclair, Completely bounded multilinear maps and $C^{*}$-algebraic cohomology, Invent. Math. 90 (1987), 279-296.

9. E. Christensen and A. M. Sinclair, A survey of completely bounded operators, Bull. London Math. Soc. 21 (1989), 417-448.

10. M. J. Crabb and A. M. Davie, Von Neumann's inequality for Hilbert space operators, Bull. London Math. Soc. 7 (1975), 49-50.

11. E. G. Effros, On multilinear completely bounded module maps, Contemp. Math. 62 (1987), 450-479.

12. E. G. Effros and E. C. Lance, Tensor products of operator algebras, Adv. in Math. 25 (1977), $1-34$.

13. J. Froelich and H. Salas, The $C^{*}$-algebra generated by a $C^{*}$-universal operator, J. Integral Equations Operator Theory (to appear).

14. D. Hadwin, Continuous functions of operators, a functional calculus, Indiana Univ. Math. J. 27 (1978), 113-125.

15. V. I. Paulsen, Completely bounded maps and dilations, Pitman Research Notes in Math., Longman, London, 1986. 
16. V. I. Paulsen and S. C. Power, Tensor products of operator algebras, Rocky Mountain J. Math. 20 (1990), 331-350.

17. V. I. Paulsen and R. R. Smith, Multilinear maps and tensor norms on operator systems, J. Funct. Anal. 73 (1987), 258-276.

18. G. K. Pedersen, $C^{*}$-algebras and their automorphism groups, Academic Press, London, 1979.

19. Z-J. Ruan, Subspaces of $C^{*}$-algebras, J. Funct. Anal. 76 (1988), 217-230.

20. B. Sz-Nagy, Sur les contractions de l'espace de Hilbert, Acta Sci. Math. 15 (1953), 87-92.

21. M. Takesaki, Theory of operator algebras. I, Springer-Verlag, Berlin, 1979.

22. N. Th. Varopoulos, On an inequality of von Neumann and an application of the metric theory of tensor products to operator theory, J. Funct. Anal. 16 (1974), 83-100.

23. J. von Neumann, Eine Spektraltheorie fur allgemeine Operatoren eines unitaren Raumes, Math. Nachr. 4 (1951), 258-281.

24. D. Voiculescu, Noncommutative random variables and spectral problems in free product $C^{*}$-algebras, preprint.

25. F. Boca, Free products of completely positive maps and spectral sets, revised preprint.

Department of Mathematics, University of Houston, Houston, TeXas 77204-3476 